\title{
Retour sur le pouvoir des bibliothèques
}

\section{Christian Jacob}

\section{OpenEdition}

Journals

Édition électronique

URL : https://journals.openedition.org/rbnu/2854

DOI : $10.4000 /$ rbnu. 2854

ISSN : 2679-6104

\section{Éditeur}

Bibliothèque nationale et universitaire de Strasbourg

\section{Édition imprimée}

Date de publication : 1 mai 2012

Pagination : 66-71

ISSN : 2109-2761

\section{Référence électronique}

Christian Jacob, «Retour sur le pouvoir des bibliothèques », La Revue de la BNU [En ligne], 5 | 2012, mis en ligne le 01 mai 2012, consulté le 25 août 2021. URL : http://journals.openedition.org/rbnu/2854 DOI : https://doi.org/10.4000/rbnu.2854

Ce document a été généré automatiquement le 25 août 2021.

\section{(c) (i) (2) (2)}

La Revue de la BNU est mise à disposition selon les termes de la Licence Creative Commons Attribution - Pas d'Utilisation Commerciale - Partage dans les Mêmes Conditions 4.0 International. 


\title{
Retour sur le pouvoir des bibliothèques
}

\author{
Christian Jacob
}

1 Quel est le statut, quelle est la nature de ce lieu particulier qu'est une bibliothèque ? Quel est son rapport à l'espace, comment organise-t-elle son espace propre?

2 On peut certes répondre à ces questions en étudiant l'ancrage géographique des bibliothèques, leur répartition sur un territoire, dans une ville, leur hiérarchie et leur complémentarité sur différentes échelles spatiales - locale, régionale, nationale, voire internationale. Une telle approche permettra de distinguer différentes formes de bibliothèques, leurs publics, leurs fonctions, leurs spécialisations, selon leur implantation.

3 L'étude des bâtiments éclairera la dimension matérielle et architecturale des bibliothèques, les formes de rationalité qui organisent les magasins, les salles de lecture, les bureaux des conservateurs et de l'administration, les espaces de circulation. Leur monumentalité et leurs programmes décoratifs (fresques, reliefs, statuaire) contribuent à la mise en exposition des savoirs et de la mémoire écrite.

4 Toute bibliothèque, en effet, est aussi un lieu de savoir, matérialisant une certaine idée de la connaissance, de la transmission, de l'articulation des disciplines, des liens entre passé et présent, de la mémoire et de la culture. Qu'il s'agisse de bibliothèques personnelles, de bibliothèques de laboratoires, de bibliothèques d'universités ou de lecture publique, ou encore des grandes institutions patrimoniales, ces lieux donnent corps et volume aux savoirs et aux arts, par les choix qui président aux contours des collections comme par les principes a priori qui les ordonnent par une hiérarchie de subdivisions.

C'est sur une autre dimension de cet espace symbolique que je voudrais réfléchir ici : certaines très grandes bibliothèques, en effet, sont comme des lieux magnétiques qui attirent, accumulent et centralisent livres, savoirs et parfois même savants, depuis une périphérie plus ou moins étendue. Ces bibliothèques produisent de l'universalité, en résorbant l'hétérogénéité et les différences locales dans la mise en ordre de la collection. Le propre de la collection est d'instaurer une synchronie, un ordre cohérent 
à des objets de provenances et d'époques diverses. Les effets produits par une bibliothèque ne se réduisent pas à la somme des effets de chacun de ses livres. La bibliothèque a un sens, une portée, un pouvoir qui dépassent ceux des livres et des textes qu'elle renferme.

6 Je voudrais illustrer cette proposition en revenant à une bibliothèque qui plus que d'autres a matérialisé le rêve d'universalité, dans les mondes occidentaux et orientaux, au fil de l'histoire et jusqu'à notre époque contemporaine : la bibliothèque du Musée d'Alexandrie. Quel est le lieu, quel est l'espace institué par cette collection? Quel est son horizon géographique?

\section{Bibliothèques clonées, bibliothèques déracinées}

7 La tradition antique souligne le rôle d'un philosophe et homme politique athénien dans la création du Musée d'Alexandrie et la constitution de sa bibliothèque : Démétrios de Phalère fut en effet le conseiller de Ptolémée $\mathrm{I}^{\mathrm{er}}$, le fondateur de la dynastie des Lagides. Le modèle de cette institution savante et lettrée était le Péripatos, l'école d'Aristote à Athènes. Comme cette dernière, le Musée réunirait une collection de livres, pour l'usage des savants, mais aussi pour le prestige du souverain et de sa capitale.

Le géographe Strabon, dans un récit célèbre consacré aux tribulations de la bibliothèque $\mathrm{d}$ 'Aristote, donne cette indication surprenante : «Aristote, le premier à notre connaissance à avoir réuni une collection de livres et avoir appris aux rois d'Égypte la manière d'ordonner une bibliothèque $»^{1}$. Ce qui passe d'Athènes à Alexandrie, ce n'est pas la collection de livres d'Aristote, mais son principe d'organisation (suntaxis), sa structure intellectuelle, l'ordre a priori dans lequel tous les livres pourront s'intégrer. Ce principe d'ordre est ce qui transforme une collection d'objets matériels en bibliothèque. Strabon ne précise malheureusement pas la nature de cette "syntaxe"; sans doute s'agissait-il de subdivisions en genres littéraires et champs de savoir qui pouvaient déterminer le rangement matériel des livres.

Le témoignage de Strabon invite à réfléchir sur la manière dont la bibliothèque d'Alexandrie a pu dupliquer celle du Péripatos, non par le contenu de la collection, mais par sa structure. Cette dernière étant un concept, un schéma intellectuel pouvant être dupliqué, reproduit et exporté, il est alors permis de dire qu'Aristote a été le maître en bibliothéconomie des Lagides, bien qu'il fût mort à l'époque de la fondation du Musée d'Alexandrie. 


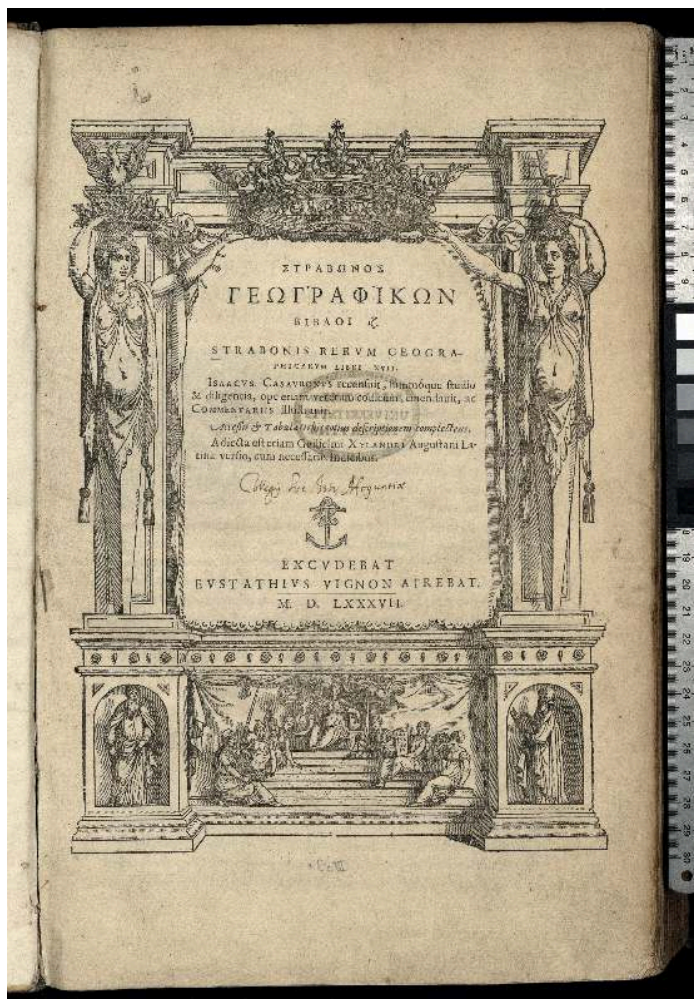

Cliché JPR-Bnu CC-BY-NC-SA

10 Les principes de classification des livres rendent les bibliothèques commensurables et comparables, quelle que soit leur échelle. Aujourd'hui, la classification de la Bibliothèque du Congrès régit des collections de livres dans toutes les régions du monde, par un découpage systématique en vingt-et-une grandes catégories qui ellesmêmes se déclinent en un grand nombre de subdivisions. Les collections peuvent différer par leur importance et leurs spécialités, elles peuvent n'utiliser que certaines subdivisions de ce modèle sans descendre tous les degrés de l'arborescence, elles s'intègrent néanmoins dans le même ordre englobant, elles reproduisent la même carte. Le lecteur qui connaît le principe global comme les subdivisions de son champ d'intérêt peut se repérer sans trop de mal dans toutes les bibliothèques qui partagent cette norme, quelle que soit leur importance.

Si la classification d'une bibliothèque peut être projetée d'un lieu à l'autre, sans que ses collections n'en pâtissent, la situation est différente lorsque c'est le fonds de livres luimême qui est déplacé. La bibliothèque d'Aristote, à nouveau, en porte témoignage. Elle fut léguée à Théophraste, qui devait prendre la succession d'Aristote à la tête du Péripatos. Théophraste la légua à son tour dans son testament à Nélée de Scepsis, mais ce dernier ne fut pas choisi pour prendre la direction de l'école. Propriétaire légitime de la collection de livres, plein de dépit, il quitta Athènes pour regagner sa patrie, Scepsis, une ville de Mysie, sur le rivage d'Asie Mineure, au nord de la Troade. Le nouveau scholarque, Straton, se trouvait à la tête d'une communauté philosophique qui venait de perdre sa bibliothèque de travail, où se trouvaient les traités de son fondateur et sa mémoire intellectuelle. Les descendants de Nélée, quant à eux, après avoir longtemps dissimulé les livres dans une cache souterraine, les cédèrent finalement à un collectionneur bibliophile, Apellicon de Téos, qui les emporta à Athènes² 
12 Les premières grandes bibliothèques romaines ont été constituées par le butin prélevé par les généraux sur les villes ou les royaumes conquis. Scipion aurait ramené à Rome les œuvres de Magon, prélevées dans les bibliothèques de Carthage ${ }^{3}$. Paul-Émile, après la victoire de Pydna, s'empara de la bibliothèque du roi Persée et la fit porter à Rome, pour ses fils ${ }^{4}$. Isidore de Séville indique d'ailleurs que c'est la première fois que l'on vit arriver à Rome une telle quantité de livres 5 . Après le saccage d'Athènes en 86 , Sylla s'empare de la collection d'Apellicon de Téos, dans laquelle se trouvaient les précieux traités aristotéliciens achetés aux héritiers de Nélée ${ }^{6}$. Enfin, en 66 avant J.-C., Lucullus ramène à Rome la bibliothèque de Mithridate VI, roi du Pont ${ }^{7}$.

\section{Microcosme / Macrocosme}

13 La Lettre d'Aristée ${ }^{8}$ est le point de départ d'une longue et riche tradition sur la traduction du Pentateuque en grec par les Septante ainsi que sur la vocation universelle de la bibliothèque d'Alexandrie. On y trouve une lettre de Démétrios de Phalère à Ptolémée Philadelphe. Le roi se préoccupait de la manière dont on pouvait encore enrichir la bibliothèque. Démétrios répond qu'il faut compléter la collection de livres grecs avec les livres de tous les autres peuples, et qu'il faut commencer par la traduction de la loi des Juifs. La réalité de cet échange est peu probable, Démétrios ayant été le conseiller du père de Philadelphe, Ptolémée I ${ }^{\text {er }}$ Lagos.

Épiphane, évêque de Salamine au $4^{\mathrm{e}}$ siècle de notre ère, est l'un des nombreux auteurs qui ont repris et enrichi le récit de la Lettre d'Aristée. Il suit la tradition de cette dernière en attribuant à tort la fondation de la bibliothèque du Brouchion, le quartier du palais royal à Alexandrie, à Ptolémée Philadelphe. Celui-ci en confie la responsabilité à Démétrios de Phalère et lui donne l'ordre de rassembler des livres de toutes les régions du monde, en écrivant des lettres à tous les rois et dirigeants de la terre et en les priant instamment de ne pas hésiter à lui envoyer des livres de poètes, logographes, rhéteurs et sophistes, iatrosophistes et historiens, etc. Comme cette opération avançait et que les livres arrivaient de partout, le roi demanda un jour au responsable de la bibliothèque combien d'ouvrages se trouvaient déjà réunis. Démétrios répondit qu'il y en avait déjà environ 54 800. "Mais nous avons appris qu'il y en a un nombre plus grand encore dans le monde entier, chez les Ethiopiens et les Indiens, les Perses, les Élamites et les Babyloniens, les Assyriens et les Chaldéens, les Phéniciens, les Syriens et les Grecs, et les Romains qui n'étaient pas encore appelés Romains, mais Latins $»^{9}$.

Grâce à la bibliothèque royale, Alexandrie devenait pour ainsi dire le centre de la terre, un lieu où pouvaient se rassembler tous les livres, les savoirs et les sagesses, de tous les horizons et en toutes les langues, des trois continents du monde connu des Anciens. Le texte d'Épiphane invite en effet à réfléchir sur les différentes logiques qui permettent à une bibliothèque de réaliser un rêve d'universalité et de complétude. Les lettres envoyées aux rois et autres dirigeants semblent circonscrites à l'espace hellénophone, peut-être aux royaumes hellénistiques nouvellement institués et aux cités grecques d'antique mémoire : elles esquissent une classification des livres par champs de savoir, par genres discursifs, déployant le programme intellectuel et littéraire de la bibliothèque. Démétrios, quant à lui, dessine un horizon plus lointain, dans l'espace comme dans le temps, en mentionnant des cultures lettrées situées aux confins du monde habité (Inde, Éthiopie) ou dans des terres d'antiques civilisations (Perse, 
Babylone, Chaldée, Phéniciens...). Il est d'ailleurs un angle mort dans ce tour d'horizon : l'Égypte elle-même, dont les monuments, l'écriture, les antiques traditions fascinaient pourtant les Grecs depuis Hérodote.

La bibliothèque d'Alexandrie accorda-t-elle réellement une telle part aux littératures des mondes non grecs? Nous ne savons pas quel crédit accorder à Épiphane de Salamine, et nous ignorons les sources anciennes sur lesquelles il a pu s'appuyer. Nous n'avons, à ma connaissance, pas d'indice que les lettrés du Musée d'Alexandrie aient fait l'effort d'apprendre des langues et des écritures étrangères. La bibliothèque d'Alexandrie a-t-elle commandité des traductions en grec de tous ces livres? Nous connaissons la traduction du Pentateuque juif, une allusion de Pline se réfère peut-être à celle des versets de Zoroastre ${ }^{10}$. Nous savons aussi que le prêtre égyptien Manéthon renouvela la connaissance grecque de l'histoire de l'Égypte, en acceptant de traduire en grec et de réunir les sources égyptiennes. Bérose avait fait de même à Babylone. La langue et l'écriture grecques étaient les seules voies d'accès des Grecs aux savoirs des autres, aux sagesses barbares, selon la belle expression d'Arnaldo Momigliano ${ }^{11}$. La traduction était le seuil obligé pour que tous les savoirs du monde entrent dans la bibliothèque d'Alexandrie.

Le Musée d'Alexandrie accueillait savants et lettrés jugés dignes d'approcher la cour royale des Lagides. Nous n'avons que des informations lacunaires sur les modalités de recrutement: de multiples réseaux entraient en ligne de compte, comme en témoigne Ératosthène de Cyrène, qui fut démarché par les envoyés des Lagides alors qu'il poursuivait des études de philosophie à Athènes. Prendre la tête de la bibliothèque d'Alexandrie était une offre qui ne pouvait pas se refuser... L'ambition universelle de la dynastie, de sa capitale et de la bibliothèque se reflétait dans le recrutement du Musée : on y trouvait des lettrés et des savants de Cyrène, de Cos, de Samos, placées sous l'influence lagide, mais aussi de Byzance et de Rhodes. Le Musée était à l'image de la ville d'Alexandrie, un monde en soi, à la croisée de multiples routes maritimes ou caravanières: vétérans de l'armée macédonienne, Ethiopiens, Nubiens, Égyptiens, Perses, Juifs, Grecs du continent, des îles, d'Asie Mineure ou de la mer Noire...

\section{Livres et textes}

Dès lors que des enjeux politiques sous-tendent la constitution d'une bibliothèque d'État, se pose la question des livres qui risquent d'échapper à cette volonté centralisatrice, à ce monopole. Dans le monde grec, la bibliothèque du Musée d'Alexandrie est la première à affirmer une ambition universelle : pallier les limites d'un pouvoir territorial limité, l'Égypte et ses zones d'influence, par une politique volontariste visant à s'approprier l'héritage d'Alexandre le Grand, non dans l'extension de l'Empire perse dont il s'était emparé, mais dans l'ambition universaliste qui avait inspiré ses conquêtes...

19 Une bibliothèque universelle est obsédée par les lacunes et les failles, par les livres inconnus qui pourraient échapper à la collection et être découverts par une bibliothèque concurrente. L'enjeu est intellectuel et symbolique autant que politique : des textes peuvent-ils circuler sans être présents dans la bibliothèque royale? Les empereurs chinois, comme les souverains lagides, se posèrent cette question. Les premiers lancèrent périodiquement de grandes campagnes de confiscation de livres pour restaurer leurs bibliothèques détruites, pillées ou incendiées. Les Lagides prirent 
une mesure douanière particulière: tous les bateaux faisant escale dans le port d'Alexandrie devaient déclarer les livres qui se trouvaient à bord. Tout rouleau de papyrus devait être remis aux agents de l'administration lagide. Des copistes se mettaient aussitôt au travail et ils produisaient dans les meilleurs délais un double du texte qui leur était confié. La copie était remise au propriétaire du livre, tandis que le rouleau de papyrus original entrait dans la bibliothèque du Musée.

20 Le médecin Galien, au $2^{\mathrm{e}}$ siècle de notre ère, est notre source sur cette politique particulière du port d'Alexandrie ${ }^{12}$. On peut y voir le cynisme et l'arrogance du pouvoir lagide, comme la manifestation d'une obsession bureaucratique et administrative : on remet au propriétaire légitime un papyrus flambant neuf, avec une copie de son texte sans doute faite à la hâte. Le livre était remis à neuf, et le lecteur pouvait penser qu'il avait gagné au change. L'exemplaire original, le rouleau originaire, provenant d'une cité lointaine et après une longue traversée maritime, terminait son voyage dans la bibliothèque du Musée. Deux logiques contradictoires se croisent ainsi. Pour le propriétaire légitime du livre, qui se voyait remettre un rouleau neuf, ce qui importait était le texte, non le support. Pour la bibliothèque du Musée, en revanche, inclure dans ses collections un livre venant d'un lieu identifié du monde grec permettait de s'approprier non seulement un texte, mais aussi ce qui le rendait singulier, son support, son écriture, sa matérialité, son histoire et son ancienneté, son ancrage géographique en un point de l'aire culturelle de l'hellénisme.

21 Les livres confisqués aux voyageurs dans le port d'Alexandrie portaient une étiquette qui les identifiait comme les "livres des bateaux ». Peut-être le nom du propriétaire originel et sa provenance étaient-ils également identifiés. De telles "métadonnées » permettaient de distinguer les multiples exemplaires d'une même œuvre: tel était le cas en particulier pour les poèmes homériques. Les scholies se réfèrent en effet à des versions identifiées par leur provenance géographique, par leurs cités d'origine. Les textes de Chios, d'Argos, de Marseille, de Sinope, de Chypre et de Crète, parmi d'autres exemplaires désignés collectivement comme les livres "des cités", offraient un témoignage précieux et de multiples points de comparaison pour le travail de correction et d'édition philologique mené dans la bibliothèque sur les poèmes homériques: variantes, lacunes, interpolations, déplacements de vers ${ }^{13}$. Au fil de la tradition alexandrine, les scholiastes font l'inventaire des conjectures et corrections présentées par les différentes versions des poèmes homériques, celles des cités, celles produites par les savants de la bibliothèque, comme Zénodote, Aristophane et Aristarque.

Ces noms de villes rendaient perceptibles la nature panhellénique des poèmes homériques ainsi que la puissance d'attraction d'Alexandrie et de son Musée, où se trouvaient réunis des livres venant de la Méditerranée occidentale comme des rivages de la mer Noire. Ces exemplaires des cités ne correspondent sans doute pas à des éditions savantes et critiques, mais à des états particuliers du texte, témoignages de différentes traditions locales ou régionales. Ces livres de provenances si diverses ont peut-être été réunis par le biais des confiscations à bord des bateaux qu'évoque Galien. Un autre épisode, que nous trouvons également chez Galien ${ }^{14}$, témoigne de la détermination et de l'absence de scrupules avec lesquelles les Lagides ont mené leur politique d'acquisition de livres. Athènes, le foyer de la tragédie au $5^{\mathrm{e}}$ siècle, détenait un exemplaire « officiel » des œuvres d'Eschyle, Sophocle et Euripide. Il ne s'agissait pas de manuscrits autographes, mais de versions des poèmes dramatiques fixées par l'écriture, 
authentifiées, utilisées dans les représentations théâtrales de la cité. C'est l'orateur et homme politique Lycurgue qui avait fait procéder à l'établissement de ces textes, au temps de Démosthène. Ptolémée Evergète prie les Athéniens de lui prêter ces précieux rouleaux pour lui permettre de copier les textes, et il leur remet un dépôt de garantie de quinze talents d'argent. Les Athéniens consentent au prêt. Ptolémée fait procéder à la copie sur du papyrus de qualité supérieure, il garde les rouleaux anciens et rend aux Athéniens les exemplaires neufs, en renonçant à récupérer le dépôt de garantie. Les exemplaires des tragiques athéniens deviennent ainsi l'un des fleurons de la bibliothèque du Musée, mettant à la disposition des critiques et lettrés alexandrins un texte authentifié et prestigieux, aussi proche que possible des textes qui étaient récités et chantés lors des festivals dramatiques en l'honneur de Dionysos.

Cette anecdote est-elle historique? Luciano Canfora soupçonne que Galien reprenne une tradition de Pergame hostile à la bibliothèque d'Alexandrie. Si l'on examine la logique interne de son témoignage, il est clair que pour Ptolémée, la mainmise sur ce fleuron du patrimoine littéraire athénien contribuait à renforcer le prestige symbolique d'Alexandrie, lieu d'attraction, de conservation, de collection de toute la culture du monde, et en particulier de l'hellénisme, à travers la mainmise sur ses supports matériels, des livres aussi anciens que prestigieux. Par la médiation des livres, Athènes se trouvait en quelque sorte décentrée, déracinée et inscrite dans la bibliothèque d'Alexandrie.

Qu'il s'agisse d'Assurbanipal à Ninive, au $7^{\mathrm{e}}$ siècle avant notre ère, des empereurs chinois ou des califes abbassides de Bagdad, aux $8^{\mathrm{e}}-9^{\mathrm{e}}$ siècles de notre ère, l'histoire des bibliothèques royales et impériales est celle de la quête des livres, de leur captation, de leur centralisation. Le rêve universaliste prend différentes formes selon les cultures, circonscrit aux frontières d'une langue et d'une tradition, ou bien ouvert aux savoirs étrangers, aux savoirs d'autrefois. Sous forme de tablettes d'argile, de rouleaux de papyrus ou de codices de parchemin, les livres traversent les frontières et viennent de tous les horizons se rassembler dans un centre de pouvoir qui affirme sa domination réelle et symbolique par la captation des mémoires et des savoirs, et par la légitimation symbolique qui en découle. L'universalité et la complétude ne sont pas seulement des fantasmes qui se déclinent sous mille formes dans l'histoire des bibliothèques: elles prennent corps dans la politique d'acquisition de ces supports, de ces objets matériels si particuliers que sont les livres. Il n'est pas de centre sans périphérie, il n'est pas de globalité sans un horizon local, il n'est pas de totalité qui ne soit construite par un ensemble de pratiques de collection, d'addition, de transformation, de traduction.

La bibliothèque d'Alexandrie reste un lieu privilégié pour réfléchir au pouvoir des bibliothèques d'hier et d'aujourd'hui, au va-et-vient entre livres et textes, au miroir qui permet à un horizon géographique, temporel, culturel et symbolique de se refléter dans un lieu de savoir et de mémoire. 


\section{NOTES}

1. Strabon, Géographie, 13, 1, 54

2. Strabon, loc. cit.

3. Pline l'Ancien, Histoire naturelle, XVIII, 22

4. Plutarque, Vie de Paul Émile, 28

5. Isidore, Etymologies, VI, 5, 1

6. Plutarque, Vie de Sylla, 26

7. Cicéron, De finibus, III, 7-8 ; Plutarque, Vie de Lucullus, 42

8. La date et l'auteur de ce texte sont discutés. Il fut écrit probablement au $2^{\mathrm{e}}$ siècle avant J.-C. par un auteur juif de la diaspora alexandrine. Le texte a été édité, traduit en français et commenté par André Pelletier, Lettre d'Aristée à Philocrate, Paris, Cerf, 1976. L'ensemble de cette tradition est étudié par Luciano Canfora, Il viaggio di Aristea, Bari, Laterza, 1996.

9. Épiphane, De mensuris et ponderibus, PG, XLIII, p. 252; je suis L.Canfora qui adopte une correction du texte grec proposée par Denis Pétau (1622).

10. Pline l'Ancien évoque le travail d'Hermippe, un élève de Callimaque, qui aurait réalisé l'index des deux millions de vers de Zoroastre (Histoire naturelle, XXX, 4).

11. Arnaldo Momigliano, Sagesses barbares, Paris, Gallimard, 1991

12. Galien, Commentaire des Epidémies, Kühn, XVII, 1, pp. 606-607

13. Les témoignages sont réunis et discutés dans l'ouvrage ancien de Thomas W. Allen, Homer, The Origins and the Transmission, Oxford, 1924, p. 283-296.

14. Galien, Commentaire des épidémies, ibid.

\section{AUTEUR}

\section{CHRISTIAN JACOB}

Directeur de recherche au CNRS, directeur d'études à l'EHESS 